\title{
ROUND THE CORNER
}

Antidepressants plus benzodiazepines in major depressive disorder: a clinical dilemma with no recent answers from research

Commentary on...Cochrane Corner

https://www.cochranelibrary.com/cdsr/doi/10.1002/14651858.CD001026.pub2/full

Angharad de Cates*, Riccardo De Giorgi

University of Oxford, Department of Psychiatry, Warneford Hospital, Oxford, OX3 7JX, UK

*angharad.decates@psych.ox.ac.uk

\section{Biography}

Angharad de Cates is a Wellcome Trust Doctoral Training Fellow at the Department of Psychiatry, University of Oxford, and an Honorary Specialist Registrar at Oxford Health NHS Foundation Trust. She is also the RCPSych Neuroscience Champion for the West Midlands.

Riccardo De Giorgi is a Wellcome Trust Doctoral Training Fellow (DPhil in Biomedical and Clinical Sciences) at the Department of Psychiatry, University of Oxford, and Honorary MRCPsych Clinical Fellow at Oxford Health NHS Foundation Trust.

They both work on experimental medicine trials in mood disorders, and have interests including the evidence-based treatment of mental illness and the neuroscientific underpinnings of psychopharmacology.

\section{Summary}

Comorbid anxiety symptoms are common in depression, and adding benzodiazepines to antidepressant treatment may seem a rational clinical solution. They also have potential to reduce the initial anxiety that may be caused by early antidepressant treatment due to their inhibitory effect via GABA-A receptor binding. This month's Cochrane Corner review examines the evidence behind combination treatment versus antidepressants alone in major depressive disorder, both in terms of the clinical and neuroscientific context. The review 
provides evidence that in the first 4 weeks of treatment, additional medication with a benzodiazepine may lead to greater improvements than antidepressant alone in terms of ratings of severity, response rates and remission rates, but not for measures of anxiety.

\section{Declaration of interest}

None.

Antidepressants and benzodiazepines: current practice, evidence, and issues

Depression is a common and disabling disorder with more than 250 million people affected globally (Malhi and Mann, 2018). Anxiety and depression often co-occur in individuals at different times as well as simultaneously, where each contributes variable amounts to the overall disease burden (Wetzler and Katz, 1989). Antidepressants are the mainstay of treatment for both disorders (NICE, 2018, NICE, 2019), but there is a delay in therapeutic outcome perhaps partly due to the time taken to desensitise presynaptic $5-\mathrm{HT}_{1 \mathrm{~A}}$ autoreceptors (Duman, 2007), and antidepressant treatment can acutely increase anxiety as an early side effect (Rahn et al., 2015, Sinclair et al., 2009). Therefore, the idea of adjuncts to reduce this early anxiety and provide support while waiting for the antidepressant therapeutic effect may be wellgrounded.

Benzodiazepines are an important class of anti-anxiety and hypnotic medication. They broadly work by binding the GABA-A receptors in the central nervous system, thus reducing the excitability and communication between neurons. However, they also interact with peripheral receptors mainly involved in immunological functions (Zavala, 1997). Such pleiotropy could be relevant when considering that the neurobiological basis of depression is likely to be diverse and involve several neurotransmitter pathways (including GABA) as well as the immune system (Duman et al., 2019).

Antidepressants are known to be clinically superior to benzodiazepines alone in treating major depression (Birkenhager et al., 1995), but national guidelines in the United Kingdom suggest a role for benzodiazepines as a combination therapy for a time-limited period if anxiety or insomnia are also present (NICE, 2018). In other words, patients with depression and comorbid anxiety, and potentially also insomnia, may particularly benefit from co-prescription 
of a benzodiazepine, particularly to relieve symptoms of anxiety and poor sleep. From a psychopharmacological perspective, such a combination appears reasonable, since most currently available antidepressants act at the level of the monoaminergic system, whilst benzodiazepines would mediate different effects on the GABAergic system. Indeed, supplementing antidepressant with benzodiazepine treatment has the potential to immediately reduce anxiety symptoms via the enhancement of action at GABA-A (Leonard, 1993). However, benzodiazepines have their own issues. Even when used at controlled doses, benzodiazepines desensitise and downregulate GABA receptors and sensitise the excitatory (glutamate) neurotransmitter system, thus resulting in tolerance and potential dependence syndrome (Allison and Pratt, 2003). Moreover, the development of tolerance may thwart longer-term benefits (Schweizer and Rickels, 1998), although this may not include anti-anxiety effects. There is also a possible increased risk of falls and motor vehicle accidents (Neutel, 1995), potentially due to detrimental effects on cognition, alertness, and motor skills.

Uncertainty regarding the mixed nature of evidence for the use of benzodiazepines as an adjunct in depression provided the motivation for the first Cochrane review on this topic, published in 2001 (Furukawa et al., 2001); this was updated in 2005, and then again in 2019 (Ogawa et al., 2019). In 2001, nine studies were included with 679 patients; in 2005, ten studies were included with 731 patients.

\section{Summary of the Cochrane review}

The Cochrane review (Ogawa et al., 2019) authors conducted a systematic review of randomised control trials where either antidepressants or antidepressants plus benzodiazepines had been randomly allocated to individuals with major depression. Ten studies published between 1978 and 2002 including 731 participants were included in the meta-analysis. Therefore, this review uses the same data as the 2005 review with minor changes in methodology. Overall, combined antidepressant and benzodiazepine therapy was more effective and tolerable than antidepressants alone in the early phase, but these effects were not maintained in later phases.

Updated methods... 
The study population included adults with major depression as defined by diagnostic criteria according to the main classification systems (such as DSM, ICD, or the Research Diagnostic Criteria) who were part of a randomised controlled trial comparing antidepressants versus combined antidepressant and benzodiazepine treatment. Considering the considerable overlap between anxiety and depression, it was particularly important that participants with comorbid anxiety disorders were included. Participants in studies with other comorbid physical or psychological disorders were not excluded automatically, unless the comorbidity itself was the focus of the study. Allowing participants with disorders outside depression to be included increases the potential generalisability of the review to provide findings applicable to typical clinical populations, but as the study population becomes less uniform it can introduce bias and reduce scientific integrity. There is a lack of clarity regarding concurrent medications: for example, were participants excluded if they were also taking other non-psychiatric medications which may potentially affect mental state, such as anti-inflammatories and steroids? This uncertainty also extends to participants potentially starting concurrent medications during the study. This sort of detail is unlikely to be recorded in the often historic study reports, and so outside the control of the Cochrane authors. However, it remains important when considering the potential applicability and clinical utility of the review, as other medications might improve or hinder the action of antidepressant medication.

The study intervention was an antidepressant plus benzodiazepine for a minimum of four weeks at a minimum effective dose according to international guidelines (we refer to the Cochrane review for details of which were included). The breadth of included antidepressants and benzodiazepines listed is a strength of the paper and improves the clinical generalisability of the findings. The European Guidelines used by the authors are not clarified, which could affect the reproducibility of their analysis. The study comparison was an antidepressant (as for intervention) but prescribed alone.

Primary outcomes were defined as effect on depressive severity and acceptability of treatment and were grouped depending on duration of administration: early ( $\leq 4$ weeks), acute (5-12 weeks), continuous (>12 weeks). Secondary outcomes, for which we refer to the Cochrane article, are not discussed in this commentary due to space. The authors were prepared to combine data from observer-rated and self-report outcomes across studies for inclusion in the meta-analysis ( $\underline{\text { Box 1)}}$; in fact, all studies had observer-rated data available, negating the need for self-report in the analysis. 
The authors only examined the highest levels of individual study evidence: randomised controlled trials, including the relevant arms of cross-over studies. To maximise the systematic nature of the search, they searched the Cochrane Common Mental Disorders Group's Controlled Trials Register, the Cochrane Central Register of Controlled Trials, MEDLINE, Embase, PsycINFO, the World Health Organization (WHO) trials portal, ClinicalTrials.gov, reference lists, and contacted relevant principal investigators to identify any additional unpublished or ongoing studies with no restrictions for language. Despite this very comprehensive strategy, from inception to May 2019, no new data emerged as published after 2002. It is perhaps surprising that no new studies were found since the 2005 update (literature searched up to 2004). However, this might indicate that recently clinical practice may have moved away from the use of benzodiazepines in general due to the concerns about iatrogenic harm, most particularly fostering dependence.

The authors used the GRADE criteria to evaluate the quality of the evidence; the addition of this was one of the updates the authors made to the review methods in the 2019 edition. Bias was classified as "unclear" from examining the reports of most studies, likely due to the age of many of the studies involved when reporting guidelines were less prevalent, and no studies had an available protocol ( $\underline{\text { Box } 2)}$.

The authors also noted particular issues regarding attrition of participants in 9 studies, with 4 studies (Yamoaka, 1994, Feighner et al., 1979, Dominguez et al., 1984, Smith et al., 2002) being of particular concern as attrition was greater than 33\%. This may increase bias and therefore concern about the validity of the findings. However, as the dropout appeared to be similar across both arms, the authors reported that they have some confidence in their findings in this respect.

The analysis was appropriately conducted according to Cochrane review standards. For metaanalysis, the authors combined continuous outcome variables of depressive and anxiety severity using standardised mean differences (SMD) with 95\% confidence intervals (CI) and calculated the risk ratio (RR) with $95 \% \mathrm{CI}$ for dichotomous efficacy outcomes. This allowed authors to be able to synthesise data from different measures assessing the same outcome. Regarding the primary outcome of acceptability, only overall dropout rates were available for all studies. 
The implication from the authors for including both continuous measures of depressive severity and dichotomous measures of depressive response and remission related to clarity and availability. They stated that response and remission data may be more available and also easier to understand. There is no equivalent justification for assessing continuous measures of severity. The authors also combined dichotomous outcome variables at what they term "approximately the same time-point" using RRs with 95\% CIs, although to allow replication this should be better explained. They also justify using empirical data combining different definitions for response as they produce similar RRs (Furukawa et al., 2011).

\section{...similar results}

The authors found moderate-quality evidence that the combination of antidepressants plus benzodiazepines compared to antidepressants alone significantly reduced depressive symptoms severity (SMD $-0.25,95 \%$ CI -0.46 to $-0.03 ; 10$ studies, 598 participants), and improved response (RR 1.34, 95\% CI 1.13 to 1.58 ; 10 studies, 731 participants) and remission (RR 1.39, 95\% CI 1.03 to $1.90,10$ studies, 731 participants). Importantly, in all cases this was only in the early (up to four week) period. For the remainder of the results, we refer readers to the Cochrane Summary. There was no data in terms of frequency of dependence.

\section{FIGURE 1 ABOUT HERE}

The forest plots (see Figure 1 and Box 4) of the Cochrane review, demonstrating pooled data subdivided for short-acting and long-acting benzodiazepines, showed that any potential clinical benefit for early use of combination therapy in terms of depressive severity is limited to longeracting benzodiazepines. This is not clearly stated in the study summary. However, the number of studies assessing short-acting benzodiazepines was small (two), which may explain the authors not exploring this further in the report. Confidence intervals were generally broad in all analyses, thus limiting the precision of findings. Heterogeneity was moderate for several outcomes, which implies that there are substantial differences between studies and metaanalyses should be conducted with caution.

In the results section, rather than the summary, the authors state that no new studies were included in this 2019 review compared to the previous 2005 review. The authors noted that 
they had brought the review processes up to data in the most recent publication, but that this had little impact on the review findings.

Sensitivity analyses were added in 2019 to check results with and without the inclusion of selfreported data, and to check results with and without the same definition of response as a 50\% reduction of depressive scores. The authors report that results were consistent within and outside of the sensitivity analyses. This aids the reader in understanding that the decisions by authors in these areas regarding inclusion did not affect the results. Other differences in the 2019 review included addition of secondary outcomes such as remission and improved clarity in time periods into early, acute, and continuous. The justification for the addition of remission as an outcome is explained by the authors. The selection of time periods for analysis is less overt. Taking into account these changes, the findings from the review appear broadly similar.

\section{To add (or not to add) benzodiazepines to antidepressants: the dilemma stands}

The major problem with the external validity of this review update is the lack of recent evidence. Many of the background references used in the review were published prior to 2000, which likely relates to the age of the original review. This is in line with a lack of included studies in the review since the last update in 2005 (none published since 2002), indicating that the evidence in this field has not substantially changed in the last 15 to 20 years. However, clinical practice in psychiatry has changed significantly even since the most recently published study in 2002. A large epidemiological study (765 130 patients) identified that the proportion of patients with concurrent new antidepressant and benzodiazepine use increased from $6.1 \%$ in 2001 to $12.5 \%$ in 2012-2014 (Bushnell et al., 2017). This is concerning considering the apparent lack of data regarding longer-term outcomes for the combination of benzodiazepines and antidepressants, alongside the potentially positive data regarding short-term use of this combination therapy. Considering the conclusions of this review, it is possible that any clinical benefit of benzodiazepines as an adjunct in depression is limited to very early use, but this is not yet clear from the randomised data included in this review. It is possible that undertaking randomised controlled trials examining longer-term outcomes of benzodiazepines when used as combination therapy in depression is difficult in view of the potential risks of dependence / withdrawal, and cognitive and motor impairments. The authors suggest that more pragmatic randomised trials may be necessary. An alternative is to agree as a community that randomised trials may no longer be possible to help us answer this clinical question, and to systematically 
assess observational data regarding longer-term use of benzodiazepines as adjuncts in depression instead. From a neuroscientific angle, and as often occurs, exciting findings in basic science (in this case, the potentially important role of the GABAergic system in the aetiology of depression, (Luscher and Fuchs, 2015)) may fail to be translated at the human research and clinical level.

There is little evidence regarding dependence from this review, a common concern about benzodiazepines (Marsden et al., 2019), and therefore important when considering the clinical applicability of these findings. Despite the potential positive findings in terms of combination therapy and a reduction in early depressive severity in this review, it remains difficult to know whether clinicians should consider adding a benzodiazepine to an antidepressant acutely if we are unclear about the potential harms of dependence with such an intervention.

The authors suggested that longer-term trials with a pragmatic design (to ensure recruitment of more typical populations and to allow for expected variations in clinical practice) are required to improve the current evidence-base particularly in terms of the potential for dependence and withdrawal for short and longer-term prescriptions. As acknowledged by the authors, only one included study (Smith et al., 2002) followed-up individuals for longer than eight weeks and could be included in the longer-term assessment of combination treatment. High dropout rates (attrition bias, Box 3) were a major problem for most of the included trials and would be worth further exploring. This was reported in the Cochrane review's discussion but less so in the review summary.

\section{Conclusion}

Overall, this Cochrane review has likely limited applicability due to few recent studies that could be included, as well as the limited length of follow-up and quality for included studies, and the few studies examining particular areas of concern such as dependence. Considering that the clinical issue underpinning the review remains as pertinent as ever, we need to remain open to exploring alternative methods of research, such as pragmatic randomised studies, observational data, and experimental medicine.

\section{Acknowledgments}


None

Boxes and figures captions:

$\underline{B o x} 1$

Self-reported outcomes: rapid and relatively easy to obtain, but subject to several biases. For example, participants may exaggerate/minimise responses because of their subjective state at the time of assessment, to receive the promised service once enrolled in the study, or for a natural tendency to respond in a way that is viewed favourably by others. Participants may also forget/misinterpret details of their clinical history and presentation (recall bias).

Observer-rated outcomes: more time-consuming, but arguably more objective than selfreported measures. Some researchers dispute the latter, as these outcomes still rely on the subject's memory and current circumstances, whilst also introducing potential biases related to the observer's experience and assumptions.

Combining data from different forms of report (for example, self-reported and observer-rated questionnaires) increases the available data for pooling but should be performed only when it is known that changes in effect sizes across studies are comparable. Even when performed carefully, results from combined outcomes can be contended by regulatory agencies.

\section{Box 2}

Study protocol: a structured document describing all the aspects of a research study. Preregistering/publishing a study protocol (for example on clinicaltrials.gov and BMJ Open respectively) has become increasingly important and several major medical journals do not accept any more studies that have not been previously registered. Pre-registered/published protocols ensure that authors pre-specify methods and analyses to prevent changing these in the context of results (selective-reporting bias), thus increasing transparency of research.

\section{$\underline{\text { Box } 3}$}

Attrition bias: due to participants leaving a study (dropouts) regardless of the reason. Dropouts may be due to chance and be randomly distributed amongst study groups; however, systematic differences between participants leaving the study and those who continue can introduce bias. Attrition always occurs to some extent: it is difficult to control but can be accounted for at the 
analysis level (for example through intention-to-treat analysis). Studies with longer follow-ups are more likely to incur into significant attrition; unfortunately, this often happens

\section{$\underline{\text { Box } 4 \text { (refers to Figure 1) }}$}

Forest plot: named after its graphs resembling a forest. Forest plots are the most commonly used way to represent the results of a meta-analysis. Article's readers may gauge the most significant meta-analysis' results by just glancing at the forest plots.

The figure represents a forest plot showing the findings over 5 fictitious studies measuring the number of dropouts (a common measure of treatment's acceptability) in people taking antidepressants (ADs) versus antidepressants plus benzodiazepines (ADs+BDZ).

Each study included in the meta-analysis is usually reported with the first author's name and date of publication (Study or Subgroup column). The comparison and intervention columns then follow - in this example, ADs and ADs+BDZ. For each of these columns, the number of outcomes of interest (Events) and the number of participants per group (Total) is reported - in this example, for Study A 2015 there were 60 dropouts out of 100 participants in the ADs arm and 50 out of 100 participants in the ADs+BDZ arm.

Each study has a different impact on the pooled result of the meta-analysis depending on how much information it contains (Weight); the weight is calculated by the statistical software and is proportional to the total participants and the total number of events for each study - in this example, Study D 2018 has much more weight than Study C 2016 (41.2\% vs 1.4\% respectively) because the former has several more participants and counts several more events than the latter.

Finally, an effect size measure (for example, a risk ratio, a mean difference, etc) with $95 \%$ confidence interval (CI) is computed by the statistical software for each study. The effect size and the $95 \% \mathrm{CI}$ are also illustrated by the graphs: the square-box shows the estimated effect size and the horizontal lines the 95\% CIs; also, and the larger the square-box, the greater the weight of the study. The vertical line of the graph is the line of no-effect - in this example measuring a risk ratio, the line of no-effect corresponds to 1 . If the horizontal line representing the $95 \%$ CI touches the vertical line of no-effect, the individual study result is not statistically significant.

At the bottom of the graph, a pooled effect size with 95\% CI is depicted in the diamond-box: the centre corresponds to the estimated effect size, whilst the lateral tips of the diamonds are the limits of the $95 \%$ CI. Again, if the $95 \%$ CI touches the vertical line of no-effect, the pooled estimate is not statistically significant, whereas if the diamond is placed clearly on the right or 
left of the vertical line of no-effect, either the intervention or the comparison is favoured - in this example, the combination of antidepressants and benzodiazepines is better than antidepressants alone in terms of acceptability (RR 1.10, 95\% CI 1.03 to 1.18) and the diamond-box is on the right side of the vertical line of no-effect (Favours ADs+BDZ). The pooled result is also reported numerically in the line in bold just below all the included studies. At the bottom of the forest plot, other numerical values are reported: it is important to notice the measure of heterogeneity across all the included studies - the lower, the better.

Author contributions:

Both authors devised the article. AdeC wrote the first draft of the article, apart from the boxes and figures which were drafted by RG. Both authors commented on drafts of the article.

\section{References}

ALLISON, C. \& PRATT, J. A. 2003. Neuroadaptive processes in GABAergic and glutamatergic systems in benzodiazepine dependence. Pharmacol Ther, 98, 171-95.

BIRKENHAGER, T. K., MOLEMAN, P. \& NOLEN, W. A. 1995. Benzodiazepines for depression? A review of the literature. Int Clin Psychopharmacol, 10, 181-95.

BUSHNELL, G. A., STURMER, T., GAYNES, B. N., PATE, V. \& MILLER, M. 2017. Simultaneous Antidepressant and Benzodiazepine New Use and Subsequent Long-term Benzodiazepine Use in Adults With Depression, United States, 2001-2014. JAMA Psychiatry, 74, 747-755.

DOMINGUEZ, R., JACOBSON, A., GOLDSTEIN, B. \& STEINBOOK, R. 1984. Comparison of triazolam and placebo in the treatment of insomnia in depressed patients. Current therapeutic research - clinical and experimental, 36, 856-865.

DUMAN, R. S. 2007. A silver bullet for the treatment of depression? Neuron. United States.

DUMAN, R. S., SANACORA, G. \& KRYSTAL, J. H. 2019. Altered Connectivity in Depression: GABA and Glutamate Neurotransmitter Deficits and Reversal by Novel Treatments. Neuron, 102, 75-90.

FEIGHNER, J. P., BRAUZER, B., GELENBERG, A. J., GOMEZ, E., KIEV, A., KURLAND, M. L. \& WEISS, B. L. 1979. A placebo-controlled multicenter trial of Limbitrol versus its components (amitriptyline and chlordiazepoxide) in the symptomatic treatment of depressive illness. Psychopharmacology (Berl), 61, 217-25.

FURUKAWA, T. A., AKECHI, T., WAGENPFEIL, S. \& LEUCHT, S. 2011. Relative indices of treatment effect may be constant across different definitions of response in schizophrenia trials. Schizophr Res, 126, 212-9.

FURUKAWA, T. A., STREINER, D. L. \& YOUNG, L. T. 2001. Antidepressant plus benzodiazepine for major depression. Cochrane Database Syst Rev, Cd001026.

LEONARD, B. E. 1993. Commentary on the mode of action of benzodiazepines. Journal of Psychiatric Research, 27, 193-207. 
LUSCHER, B. \& FUCHS, T. 2015. GABAergic control of depression-related brain states. Adv Pharmacol, 73, 97-144.

MALHI, G. S. \& MANN, J. J. 2018. Depression. Lancet, 392, 2299-2312.

MARSDEN, J., WHITE, M., ANNAND, F., BURKINSHAW, P., CARVILLE, S., EASTWOOD, B., KELLEHER, M., KNIGHT, J., O'CONNOR, R., TRAN, A., WILLEY, P., GREAVES, F. \& TAYLOR, S. 2019. Medicines associated with dependence or withdrawal: a mixedmethods public health review and national database study in England. Lancet Psychiatry, 6, 935-950.

NEUTEL, C. I. 1995. Risk of traffic accident injury after a prescription for a benzodiazepine. Ann Epidemiol, 5, 239-44.

NICE 2018. Depression in adults: recognition and management. London.

NICE 2019. Generalised anxiety disorder and panic disorder in adults: management. London.

OGAWA, Y., TAKESHIMA, N., HAYASAKA, Y., TAJIKA, A., WATANABE, N., STREINER, D. \& FURUKAWA, T. A. 2019. Antidepressants plus benzodiazepines for adults with major depression. Cochrane Database Syst Rev, 6, Cd001026.

RAHN, K. A., CAO, Y. J., HENDRIX, C. W. \& KAPLIN, A. I. 2015. The role of 5-HT1A receptors in mediating acute negative effects of antidepressants: implications in pediatric depression. Transl Psychiatry, 5, e563.

SCHWEIZER, E. \& RICKELS, K. 1998. Benzodiazepine dependence and withdrawal: a review of the syndrome and its clinical management. Acta Psychiatr Scand Suppl, 393, 95-101.

SINCLAIR, L. I., CHRISTMAS, D. M., HOOD, S. D., POTOKAR, J. P., ROBERTSON, A., ISAAC, A., SRIVASTAVA, S., NUTT, D. J. \& DAVIES, S. J. 2009. Antidepressant-induced jitteriness/anxiety syndrome: systematic review. Br J Psychiatry, 194, 483-90.

SMITH, W. T., LONDBORG, P. D., GLAUDIN, V. \& PAINTER, J. R. 2002. Is extended clonazepam cotherapy of fluoxetine effective for outpatients with major depression? J Affect Disord, 70, 251-9.

WETZLER, S. \& KATZ, M. M. 1989. Problems with the differentiation of anxiety and depression. J Psychiatr Res, 23, 1-12.

YAMOAKA, K. 1994. Augmentation therapy of antidepressant and benzodiazepine in treatment of depression. Seishinka-chiryo-gaku, 9, 1349-1355

ZAVALA, F. 1997. Benzodiazepines, anxiety and immunity. Pharmacol Ther, 75, 199-216.

Author Details:

Angharad de Cates, BMBCh (Hons), BA, MSc, MRCPsych, Welcome Trust Clinical Doctoral Fellow, Department of Psychiatry, University of Oxford, and Honorary Specialist Registrar, Oxford Health NHS Foundation Trust, United Kingdom.

Riccardo De Giorgi, MD (Milan), MRCPsych, Welcome Trust Clinical Doctoral Fellow, Department of Psychiatry, University of Oxford, and MRCPsych Clinical Fellow, Oxford Health NHS Foundation Trust, United Kingdom 


\section{Figure 1:}

ADs ADs + BDZ Risk Ratio

Study or Subgroup Events Total Events Total Weight IV, Fixed, 95\% Cl

Study A 2015

Study B 2016

Study C 2016

Study D 2018

Study E 2019

Total $(95 \% \mathrm{Cl})$

Total events

Heterogeneity: $\mathrm{Chi}^{2}=40.33, \mathrm{df}=4(\mathrm{P}<0.00001) ; \mathrm{I}^{2}=90 \%$

Test for overall effect: $Z=2.66(P=0.008)$
Risk Ratio

IV, Fixed, 95\% Cl

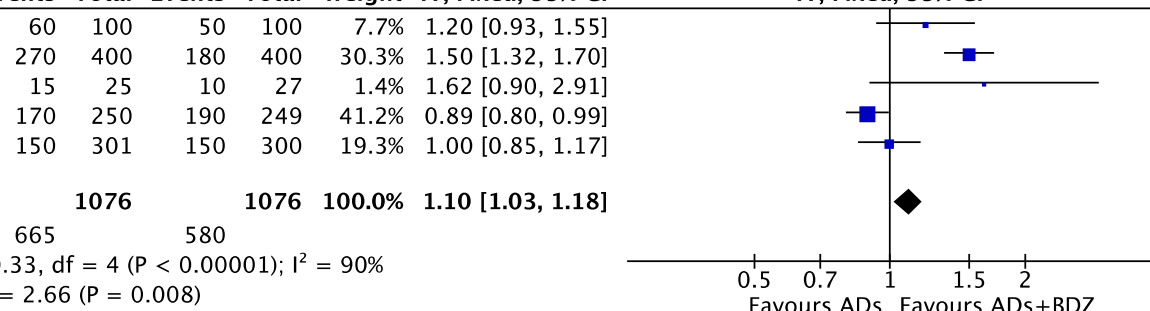

